\title{
ENERGY EFFICIENT ROUTING ALgORITHM For VOID AVOIDANCE IN UWSN USING RESIDUAL ENERGY AND DEPTH VARIANCE
}

\author{
Gulista Khan and R.K. Dwivedi \\ Department of Computer Engineering, Teerthanker Mahaveer University, \\ Moradabad, UP, India
}

\begin{abstract}
Underwater Wireless Sensor Networks (UWSN) is attracting the interest of most of the researcher because of the good opportunity to discover and catch the oceanic activities. As we know radio waves could not work efficiently in Underwater so Underwater Acoustic Sensor Networks (UASN) emerged as a most prevalent network to an outstanding range. UASN have some constraints in its deployment as well as acoustic wave communication. This limitation involves large propagation delay, transmission cost, very less bandwidth, high signal attenuation, and restricted accessibility of the nodes and non-availability of the recharging of nodes leads to the development of some energy saving algorithms to prolong the lifetime of the nodes. Routing technique must be rich enough to overcome all these constraints and give an energyefficient path by avoiding void regions and increase the network lifetime. Depth based algorithms proposed in the last decades use depth factor to estimate the path from sender to the sink. By having the holding time calculation they minimize the replication of information. Here, this paper have proposed Energy Efficient Void Avoidance Routing Scheme for UWSN (E2RV) using Residual Energy and Depth Variance it used two hop node information to escape the void shacks in the network area along with this it is using regularized remaining energy and normalized depth of the nodes to estimate the path from data generating node to sink node. In this way E2RV not only removing the void holes but also maintains the energy depletion of the network nodes and upsurge the network lifetime. Simulation results show the improvement of E2RV over previously defined algorithms in terms of packet delivery ratio, duplications, less energy depletion and increased lifetime.
\end{abstract}

\section{KEYWORDS}

Acoustic, underwater wireless device networks, void region, residual energy, and normalized depth.

\section{INTRODUCTION}

$2 / 3$ components of the world are surrounding by water (Ocean) this implies an excellent comparable risk of underwater examination. It is gaining the interest of most of the researchers because of its vast applications in military and non-military applications. The applications range from the seismic activity, marine ecosystem, water pollution, offshore exploration and coastal area surveillance. To get the benefit of these applications continuous monitoring is required through some sensors. Data Sensing and the communication to the base station for getting relevant information is a stimulating task in Underwater Acoustic Sensor Network (UASN) since, it using the acoustic sensor nodes. Acoustic sensor nodes have many constraints such as low bandwidth, high delay, high attenuation etc.

The main characteristic of UASN is sensing the data and sending this sensed data to the sink node. Sensing is done by the sensors called simple nodes having fixed transmission power and has small memory to store the sensed data. Transmission and receiving unit is it. These nodes operate on the battery. Recharging of this battery is just not possible because of the higher cost in 
getting the nodes at surface recharge it and deployed it back. Simple nodes are usually set at the bottom of the ocean or attached to the seabed through some cord.

These sensor nodes can communicate to the other nodes or sink through the various signals available for communication. Radio signals $[1,2,4,5]$, Optical signals $[6,7]$ and acoustic signal $[23,3]$. Among all these signals, underwater nodes use acoustic signals to communicate. They use sound waves for communication. In spite of having some advantages of using acoustic signals underwater it is having some limitations also. Acoustic signals have large propagation delay, less bandwidth, high attenuation, high energy consumption, large communication range, high error rate $[8,9]$. Also sink nodes are at the water surface. Sinks uses two interfaces to communicate. One Interface is used to collect the information from the sensor other is used to direct this collected information to the base station. One interface using acoustic communication second is using radio communication mode.

The UASN scenario is shown in the figure 1. Sensor nodes are organized in the network area. Trs is denoting the transmission range of the node s. Radio waves and optical communication waves can also be used for underwater communication. But these are not suitable due to various characteristics of these waves.

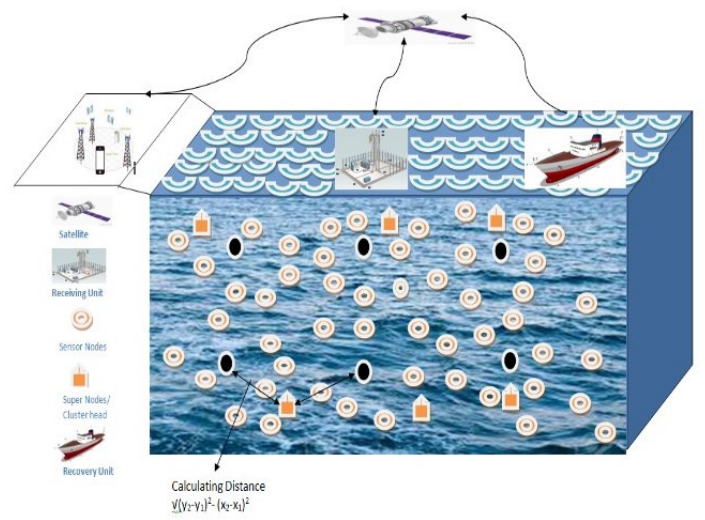

Figure 1: UWSN Environment

Radio signals are preferably used in earthy communication because of less consumption of energy, less delay in propagation, low signal attenuation. Due to this constraint only sink node is using the radio signals to communicate to the base station. Same time we cannot use radio signals to communicate among simple nodes in the aquatic environment. In this environment radio signals are not used because of high propagation delay, low bandwidth and high absorption due to absorption in water. Radio's signals will have the less transmission power in an underwater environment. In the same way we cannot use optical signals in the aquatic situation because it requires a clear streak of view between sender and the receiver but this is very difficult because of disturbance due to water currents. Since radio signals and optical signals have limitations in the aquatic environment, acoustic signals are the only option and it is widely used for communication.

There are various directing protocols published in collected works that consider the underwater communication through acoustic signals with its limitations such as large error rate, high propagation delay, low bandwidth, high energy consumption. Routing algorithms proposed in the literature are categorized into two categories, Location dependent [10-14] and Location independent [15-20]. In Location dependent routing algorithm each and every nodule knows its own position. Sometimes it knows the location of its neighbors also. By knowing locations of neighbors sender can forward the data packet towards the sink. By knowing the location sender can direct the packet towards sink through its neighbors, by doing so it assumes less propagation 
delay, consumes less energy, and lesser bandwidth. The second category of the routing algorithm is Location Independent. In this type of algorithm nodes do not know their location. Nodes can calculate their depth through the pressure applied on these nodes through the water. Also they do not have any knowledge or information of the receiver node; they only consider that data package has to be directed to the surface only. Node has information about the depth of the next hop neighbor only.

The routing algorithm constructed using the depth information, decreases the propagation delay and energy consumption. Sender node directed the data packet to the next nodes having lower depth then the sender node, if sender has multiple neighbor nodes at lower depth then it will onward the data packet to the lowest depth node. All other neighbor nodes at lower depth simply discard/Suppress the packet $[15,26]$. This phenomenon of suppression reduces the energy consumption. It is implemented by the holding time strategy. Holding time can be calculated by the knowledge of the depth information only. Nodes calculate their holding time to grasp the data packet. The node having smallest holding time selects to accelerative the data packet towards the sink. Once the node selected having smallest holding time, it starts the timer. It waits till the timer expires, if any other copy of same data packet received during this timer then it simply suppresses the packet as soon as the timer expires. It forwards the data packet towards the sink node. Nodes who have the lesser depth than the sender node will take participation in calculating the holding time. The node having lowest depth and lowest holding time selects as the forwarder for the data packet. To ensure the lesser energy consumption and shorten the propagation delay lowest depth neighbor is selected as the forwarder and it must be having lesser holding time. Some depth based algorithms not only using the depth information for routing, but considers various other factors also such as link quality, holding time, depth of next forwarder node [16-20].

Such type of depth based algorithm gives the better results in starting but penalizes the lesser depth nodes very quickly. For such type of routing every time, the same set of nodes are selected for forwarding purpose. In such ways these nodes drain out their battery power very quickly. After some rounds these nodes dead and creates the void region. The void regions are those regions where there is no node available in the region to forward the data packet. So algorithms must consider this void region and must not select the next node that falls in the void region. Because if that node does not have any node in the region then it will discard the packet. However this identification of the void region is very difficult. So the algorithm must be designed to avoid the void regions and disperse the load among other nodes that not involve in the void region. It can be done by checking the remaining energy concept. The nodes having lesser remaining energy are will not take participation in the packet forwarding. The nodes having lowest depth, lesser holding time and highest remaining energy will be considered as the forwarder node for data transmission.

By keeping all these considerations in mind, we have proposed an E2RV algorithm. E2RV calculates the next forwarder nodes, if:

I. The nodes having maximum remaining energy among its neighbor nodes.

II. It has at least one node having lesser depth.

III. It has multiple nodes having a lesser depth than the sender node.

By having these characteristics we avoid the void regions, increase the network lifetime by decreasing the energy ingestion, avoid packet collision by discarding multiple copies of the node. 


\section{LITERATURE REVIEW}

Under Water Sensor Networks (UWSNs) gives a solution to monitor those environments where human presence is difficult. As compare to earthy networks UWSNs having several limitations of low bandwidth, high propagation delay and low transmission power. "These networks are generally designed by the acoustic sensor nodes and surface sinks called buoys that are linked to any onshore control center. Some characteristics of acoustic channel generate multiple problems like, low available bandwidth and high error probability that control the efficiency of UWSNs. A part of this limited resources and node movement are main intimidations for the reliable data delivery. So it is difficult to make an algorithm that consists the ability to increase the reliability of the networks". Thus several routing algorithms based on location and location less algorithms has been reviewed. On the basis of literature survey many challenges [5] are still not addressed i.e. high propagation delay, network lifetime and lower bandwidth. Under water Sensor Network consists of a sensor module that has software modems and generic hardware to sense data acoustically in water and send collective data to the base station, as shown in Figure 2.

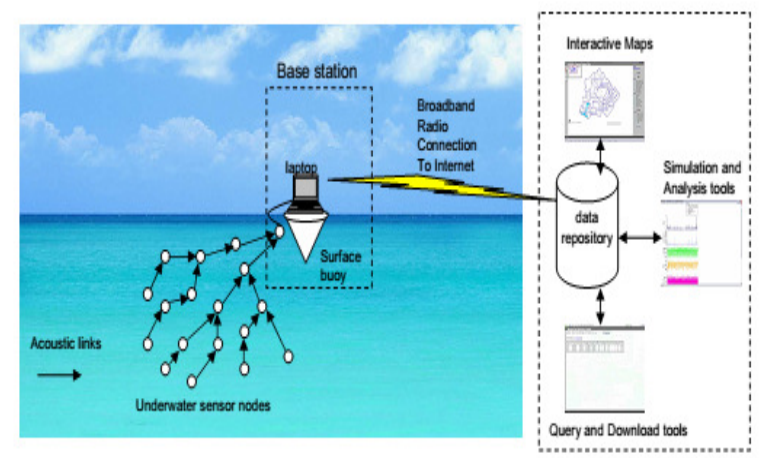

Figure 2: Under Water Sensor Network Application

In [21-23] survey on research challenges for designing routing algorithms for UWSN has been presented. Main challenges in designing the UWSN algorithm are to have limited power, low bandwidth, and high delay and void region problem. In [24] one algorithm proposed which uses back off timers to avoid the duplicate packet transmission. Back off timer is randomly set for some duration. When a node receives a data packet it starts a back off timer, it holds the transmission of that packet for that time duration. If nodes receive duplicate copies of the same packet within this time duration then it discards those copies. The algorithm proposed in [25] uses the back off timer with hop count to discard the duplicate copies. Many algorithms in past uses the same concept of timer and hop count.

As discussed in earlier section depth information is the major parameter to decide the next forwarder node to forward data to base station. Nodes at the higher depth send the data packet to the sink node through nodes at lower depths. These depth based algorithms can be categorized in two forms, Sender initiated and receiver initiated. In the sender initiated approach, senders decide the next forwarder node and add its information to the data packet. $[17,19]$ On the other hand in receiver- initiated approach, the receiver node decides whether it wants to forward the packet or not. $[15,16,18,20]$

In [15], an algorithm named as Depth Based Routing (DBR) has been proposed. It is holding time-based packet suppression technique. Consider a scenario in which a node $S$ wants to forward a packet to the sink node. Its neighbor nodes are 1,2,3,4 and 5. The nodes 1 and 5 are placed at higher depths than node $\mathrm{S}$ and nodes 2, 3 and 4 are placed at a lower depth. Now when 
node $\mathrm{S}$ sends a data packet. Nodes at the higher depths simply discard the packets i.e. nodes 1 and 5 discards packet. Now nodes 2, 3 and 4 receive the data packet and calculate their holding time. The node that is placed at the lowest depth and having higher depth difference is having shorter holding time. Shortest holding time node is selected as the next forwarder. In DBR like techniques all the data forwarding is done from lower depth nodes to higher depth nodes. In this way lower depth nodes die out earlier, cause the void regions.

Abdul Wahid et al. [16] Proposes an Energy-Efficient Depth-Based Routing algorithm (EEDBR) for UWSN, Author explains "algorithm for designing network/routing in UWSNs which is a challenging task. Also, to improve energy efficiency is an issue of concern in UWSNs, because of battery replacement in underwater is very costly due to the unpleasant condition of underwater. So the author proposes an algorithm, named EEDBR for energy efficient routing in UWSNs. Which considers the deepness of sensor nodes along with the balanced energy of nodes to find the appropriate forwarder to transmit the data back to the sink node?

Abdul Wahid et al. in [27] explain the routing algorithm for UWSN named as MRP (Multilayer routing algorithm). The proposed algorithm works on two factors. First one is acoustic signals are used to sense the data packet. The second one is this algorithm considers a superior node having unpredictable transmissions power and rich energy. To reduce the energy intake, the limited numbers of nodes are used at the time of transmission of data from source to sink node.

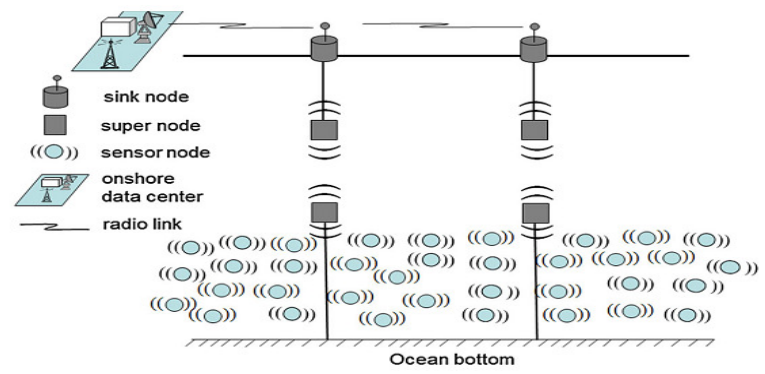

Figure 3: MRP Architecture [27]

G. Liu et al. in [28], proposed a Depth-Based Multi-hop Routing (DBMR). Since DBR uses flooding mode for transmission, a large amount of redundant data forwarding and channel occupancy might be caused. To reduce communication overhead, DBMR adopts Multi-hop to send packets. Moreover, Depth-based multi-hop routing can take advantage of multiple-sink UWSN architecture without increasing any additional cost.

Authors have proposed inherently void avoidance routing protocol (IVAR) in [18]. IVAR works on the principal of DBR along with hop count of a node. When a sender node sends a data packet to the sink node it included its hop count in the packet. Data is being forwarded from higher depth nodes towards the lower depth nodes. All the neighbor nodes having lower hop count are eligible to become the forwarder node. So all eligible nodes calculate their holding time. Lower depth nodes that are having the lower hop count and the shorter holding time is selected as the next forwarder node. Node placed at the edges of void regions simply discards the message.

Authors in [19] proposed an algorithm named Opportunistic Void Avoidance Routing (OVAR). It improves the shortcomings of the IVAR algorithm. It is sender initiated opportunistic algorithm. OVAR adapts the same beacon method to maintain information. It works on proximity to avoid the void hole problem.

A receiver initiated algorithm has been designed by the authors in [20], named as weighted depth and forwarding area division DBR (WDFAD-DBR). This works on the principal of DBR, along 
with it suppress the duplicate data packets transmission and reduce delay by taking two-hop distance value in calculating the holding time.

In [29] author says UWSN has detectors with internal restrictions because of it's deployed within the aquatic surroundings and use of water signals to speak. There are several restrictions like delay in propagation, terribly restricted information measure, not economic for transmission, terribly high peak of signal and change of power source. Thus UASN must consider these restrictions to obtain less energy consumption and loss, better time management of the network. Nodes based on depth generally carry information towards the sink. This helps in reducing the duplicity of data and time management. Hence to stay away from holes production authors proposed to use 2 hop nodes restricted information. EDOVE theme for realizing energy reconciliation and holes dodging within the network. EDOVE propagates regarding 53 less copies of the information will increase life of network; it also leads to depletion of the energy of nodes as compare to WDFAD DBR.

\section{Proposed E2Rv Algorithm}

This section presents the proposed algorithm E2RV. This removed the void regions by selecting the forwarder node having the maximum residual energy, multiple nodes having varying depth. It also considers the holding time calculation to discard the same packets. Void regions can be avoided by prioritizing the nodes based on depth, residual energy and holding time. It uses a weighting factor to calculate the two hop depth difference, i.e. either the depth difference (Di) between the senders $\mathrm{S}$ or its one-hop receiver say i, and second hop difference between (Di_nf) one hop receiver i and its next hop neighbor $\mathrm{j}$.

$$
\begin{gathered}
\mathrm{D}_{\mathrm{i}}=\left(\mathrm{d}_{\mathrm{S}}-\mathrm{d}_{\mathrm{i}}\right) \\
\mathrm{D}_{\mathrm{i} \_n f}=\left(\mathrm{d}_{\mathrm{i}}-\min \left(\mathrm{d}_{\mathrm{j}}\right)\right)
\end{gathered}
$$

Two hop depth difference is denoted by $\mathrm{H}$, where

$$
\mathrm{H}=\alpha(\mathrm{Di})+(1-\alpha)\left(\mathrm{D}_{\mathrm{i} \_\mathrm{nf}}\right)
$$

Here $\alpha$ is used to prioritizing $D_{i}$ and $D_{i \_n f}$ Its value ranges from 0 to 1 . Maximum delay in acoustic signals is termed as $\beta$, and holding time between the receiving units can be calculated by multiplication of $\mathrm{H}$ by $\beta$ :

$$
\mathrm{H}_{\mathrm{ti}}=\beta\left(\mathrm{tr}^{\mathrm{S}}-\mathrm{H}\right)
$$

Where $\operatorname{tr}^{\mathrm{S}}$ is the transmission range of sensor node $\mathrm{S}$. Here $\beta$ is depending on $\mathrm{p} \epsilon\left(0, \operatorname{tr}^{\mathrm{S}}\right)$ and calculated by

$$
\beta=\left(2 \operatorname{tr}^{\mathrm{s}} / \mathrm{v}_{\text {sound }}\right) / \mathrm{p}
$$

WDFAD-DBR uses the above formula to calculate the acoustic delay. E2RV calculates it by using the biased two hop depth variance, residual energy of node i, next hop depth dissimilarity variance $6 i$.

Hence holding time is thus given the priority to the node having the highest remaining energy, minimum holding time, large depth difference variance. In this way energy can be efficiently organized by avoiding the packet collision and avoid the void area. This in turn increases the network lifetime. 


\subsection{Problem Statement}

Consider an underwater scenario where sensor nodes are denoted by $S_{i}$, where $i=1,2 \ldots . n$. All the receivers having residual energy $E_{i}$, inside the transmission range $\operatorname{tr}^{S}$ of sensor node $S$. Sensor node generates the data packet by sensing the environment. Then it further forwards the packet towards the sink nodule through one or more hops. Hop nodules simply act as the relaying node, they hold the packet for some time then forward packet to next hop node or to the sink node. Si may have the multiple neighbors, so routing algorithm should be based on the depth information, decreases the propagation delay and energy consumption. Sender node promotes the data packet to the next nodes having lower depth then the sender node, if sender has multiple neighbor nodes at lower depth then it will promote the information packet to the lowest depth node. Along with it if the neighbor node having lower depth and higher residual energy are at the edge of the void region then we must avoid to selecting these nodes. Otherwise packet will be dropped. All other neighbor nodes at lower depth simply discard/Suppress the packet [15]. This phenomenon of suppression reduces the energy consumption. It is implemented by the holding time strategy. Holding time can be calculated by the knowledge of the depth information only. Nodes calculate their holding time to hold the data packet. The node having smallest holding time selects to further send the data packet towards the sink. Once the node selected having smallest holding time, it starts the timer. It waits till the timer expires, if any other copy of same data packet received during this timer then it simply suppresses the packet as soon as the timer expires. It forwards the data packet towards the sink node. Nodes who have the lesser depth than the sender node will take participation in calculating the holding time. The node having lowest depth and lowest holding time selects as the forwarder for the data packet. To ensure the lesser energy consumption and shorten the propagation delay lowest depth neighbor is selected as the forwarder and it must be having lesser holding time. Some depth based algorithms not only using the depth information for routing, but considers various other factors also such as link quality, holding time, depth of next forwarder node [16-20].

\subsection{Preliminary Definitions}

E2RV uses following notations:

3.2.1. Network Size $(\mathrm{N})$ : $\mathrm{N}$ is denoted the total number of nodes dispersed in the network area having the acoustic communication capabilities.

3.2.2 Receiver nodes $\left(\mathrm{Rs}_{\mathrm{i}}\right)$ : Receiver nodes are the nodes which are in the transmission and receiving a ranging of $S$ and having the lower depth than $S$.

$$
\mathrm{Rs}_{\mathrm{i}}=\left(\mathrm{i} \in \mathrm{N} I \mathrm{di}<\mathrm{ds} \wedge \mathrm{D}(\mathrm{i}, \mathrm{s})<\mathrm{tr}^{\mathrm{S}}\right)
$$

Where D (i, s) is denoting the Euclidean distance from sensor nodule to i node (neighbour node). $D_{i}$ is the depth of node and $d_{s}$ is the depth of node $s$ respectively.

3.2.3 Forwarder node NFsi: These are the nodes which lie in the broadcast range of node $\mathrm{S}$ and having the depth less than node $\mathrm{S}$.

$$
\mathrm{NFsi}=\left(\mathrm{j} \in \mathrm{N} \mid \mathrm{dj}<\mathrm{ds} \wedge \mathrm{D}(\mathrm{j}, \mathrm{s})<\mathrm{tr}^{\mathrm{s}}\right)
$$

Residual energy of node $\mathrm{Si}$ : the current level of battery power of node $\mathrm{Si}$.

3.2.4. Normalized depth discrepancy of node $\mathrm{Si}$, $6 \mathrm{i}$ : It is the normalized depth discrepancy of all the nodes in NFsi. 


\subsection{Estimation of Holding time}

Node S sense the environment and prepare a data packet. Forward this packet to the node i within its transmission range $\operatorname{tr}^{\mathrm{S}}$. Then node i checks and compare its depth to the sender if Di>Ds then it simply rejects the information packet. If its depth is less than the sender node $S$ then check the forwarder nodes set NFsi. If it is having more than one forwarder node than it calculated the holding time Hti. If all the neighbour nodes having higher depth than sender then they simply discard the information packet. The holding time of the selected forwarder nodule must be small to all the neighbour nodes, with the highest residual energy, large depth difference.

E2RV compute the holding time of $i \in N F s$ with following formula:

$$
\begin{gathered}
\mathrm{NFs}_{\mathrm{i}}=\left(2 * \mathrm{tr}^{\mathrm{s}}\right) / \mathrm{v}_{\text {sound }} *(\mathrm{H}) \\
\text { Where } \mathrm{H}=\mathrm{ei} *\left(1-\mathrm{d}_{\mathrm{i}} / \mathrm{tr}^{\mathrm{s}}\right)+\left(1-\max \left(\mathrm{d}_{\mathrm{NFj}}\right) / \mathrm{tr}^{\mathrm{i}}\right) * \sigma_{\mathrm{i}}
\end{gathered}
$$

$\mathrm{J}=1,2 \ldots \mathrm{NF}_{\mathrm{i}}$

$$
\mathrm{V}_{\text {sound }}=1500 \mathrm{~m} / \mathrm{s}
$$

Here in equation (8) we calculated the holding time. In this firstly we select the neighbours from the neighbour set with lower depth and larger depth difference with respect to the sender node. It must have high-normalized residual energy. By this, we can increase the network lifetime by doing energy stability and disseminating data in the network. Equation (4) also taking count of the depth variance and maximum depth difference among neighbour nodes.

The factor ei is the remaining energy of the nodes can be calculated by following formula:

$$
\mathrm{e}_{\mathrm{i}}=\frac{E \max -E i}{E \max -E \min }
$$

Where $\mathrm{E}_{\mathrm{i}}=$ Residual Energy of node $\mathrm{i}$

$\mathrm{E}_{\max } \neq \mathrm{E}_{\min }$

$\mathrm{E}_{\min }=\min \left(\mathrm{E}_{\mathrm{k}} \mid\right.$ for all $\left.\mathrm{k} \in \mathrm{NF}\right)$

$\mathrm{E}_{\max }=\max \left(\mathrm{E}_{\mathrm{k}} \mid\right.$ for all $\left.\mathrm{k} \in \mathrm{NF}\right)$

$$
\sigma \mathrm{i}=1-\left(\left(1 /\left(\mathrm{NF}_{\mathrm{i}}-1\right) * \sum_{i=1}^{N F i}(\operatorname{diNF}-\mu)^{2} / \operatorname{tr}^{\mathrm{i}}\right.\right.
$$

Here $\mu=\left(\left(1 /(\mathrm{NFi}-1) * \sum_{m=1}^{|N F i|}(d m)^{\mathrm{NF}}\right.\right.$

The value of $6 \mathrm{i}$ is lesser for the node $\mathrm{i}$ which has greater normalized depth dissimilarity discrepancy and this node will be selected as the next forwarder node. In this way the E2RV will have the next forwarder nodes having large remaining energy and large depth discrepancy and a smaller depth than the source node.

In computing the holding time, source node must know the distance and residual energy of all the nodes so, for this all the neighbour nodes will send this information to the sensor nodes periodically by (E_nbr, D_nbr) message. Sensor nodes collect this information in the neighbour table with field nbr_id, E_nbr, D_nbr. 


\section{Algorithm: Proposed Algorithm at the receiver node}

Input: Receiver node say i receives the sensed data packet containing $\left\{S_{-}\right.$id, ds, $E_{\max }, E_{\min }, t^{S}$, data\}

Output: Forward the packet to two hop neighbour/ sink node or Discard the packet.

Initialize packet queue as $Q_{\text {ueue }}$.

Initialize receiver node set as $\mathrm{R}_{\mathrm{i}}$.

$\mathrm{NF}_{\mathrm{i}}$ is the next forwarder node list.

Timerdata is the regulator for the data packet received.

//if data not belongs to the queue then add it to queue.

If (data $\epsilon$ ! $Q_{\text {ueue }}$ ) then

Add data to Queue

If $\left(\right.$ Timer $_{\text {data }}==$ OFF$)$ then

Node i receive the data packet. $\left\{\mathrm{ds}, \mathrm{E}_{\max }, \mathrm{E}_{\min }\right.$,

$\left.\operatorname{tr}^{\mathrm{S}}\right\}<-$ data

If $\left(i \in R_{i}\right)$ then

If $\left(\mathrm{NF}_{\mathrm{i}} !=0\right)$ then

Calculate residual energy $E_{i}$

Calculate depth variance

Calculate holding time

Set Timer $_{\text {data }}=\mathrm{Ht}_{\mathrm{i}}$

Start Timer ${ }_{\text {data }}$

Call

end

end

end

Remove data from $\mathrm{Q}_{\text {ueue }}$

Update One-hop NBRtable

Drop (datapacket)

Exit

Else

Drop datapacket

Exit

End

Call Procedure

Compute data from $\mathrm{Q}_{\text {ueue }}$.

Estimate Emax and Emin from NBRtable

Estimate depth and update $\left\{\mathrm{ds}, \mathrm{E}_{\max }, \mathrm{E}_{\min }, \mathrm{tr}^{\mathrm{S}}\right\}$

Forward datapacket

Remove data from the $\mathrm{Q}_{\text {ueue }}$

\section{Performance Analysis}

The performance comparison of different algorithms with E2RV has been discussed here. Authors have considered the 3D underwater structure of network area of $1 \mathrm{~km}$ width and length and depth of $2 \mathrm{~km}$. Nodes having homogeneous energy levels which are ranging from $600 \mathrm{~m}$ to $1000 \mathrm{~m}$. Sink node is placed at the surface of water. All other nodes are deployed at various locations as per deployment algorithm. Any node can be a sender at a particular time. Nodes act as a sender as well as the forwarder nodes. 
In the first simulation process we have deployed 60 nodes, next trial we deployed 90 and in third trial 120 nodes. Deployed five sink nodes over the water surface. Each and every node is assigned primary energy E0. Energy consumption by a node to transfer $\mathrm{k}$ bits at some distance $\mathrm{d}$ meters is defines as:

$$
\mathrm{E}_{\mathrm{x}}(\mathrm{k}, \mathrm{d})=\mathrm{E}_{\mathrm{elec}} * \mathrm{k}+\mathrm{E}_{\mathrm{amp}} * \mathrm{k} * \mathrm{~d}^{2}
$$

Where $E_{\text {elec }}$ is the power required to send one bit of data. Eamp is the power required to amplify the signal. It is known as acoustic amplifier energy. The Energy required to receive the data of $\mathrm{k}$ bits is represented by: $E_{r x}=E_{\text {elec }} * k$. As per the energy model we have calculated receiving, transmission and idle energies as, $158 \mathrm{~mW}, 50 \mathrm{~W}$, and $58 \mathrm{~mW}$ respectively.

Table 1: Parameter Setting

\begin{tabular}{|l|l|}
\hline Parameter Name & Parameter Vahe \\
\hline Ex & $50 \mathrm{~W}$ \\
\hline $\mathrm{E}_{\mathrm{r}}$ & $158 \mathrm{~mW}$ \\
\hline Ideal Energy & $58 \mathrm{~mW}$ \\
\hline Header Size & $88 \mathrm{bits}$ \\
\hline Payload Size & $576 \mathrm{bits}$ \\
\hline Neighbor request & 48 bits \\
\hline Acknowledgement & 48 bits \\
\hline Acoustic propagation delay & $1500 \mathrm{~m}$ \\
\hline Data rate & $16^{*} 10^{3}$ bits \\
\hline Packet generation rate & 0.2 packet $/ \mathrm{sec}$ \\
\hline Weighting factor $\alpha$ & 0.5 range $(0,1)$ \\
\hline
\end{tabular}

We have done this simulation more than 100 times and calculated the average results are shown in graphs.

\subsection{Broadcast Copies of Data Packets}

The first parameter we have taken is, to investigate the total quantity of broadcast copies in the network system. Figure 4 and 5 showing the number of broadcasted copies with respect to the broadcast Range and network system size. It is clear from the figure that the broadcasted copies are straight relative to the network system size. It is just because, when network size increases a large number of packet transmits. The smaller network could not cover the whole network. So when the network size increases, a large number of data transmission copies sent.

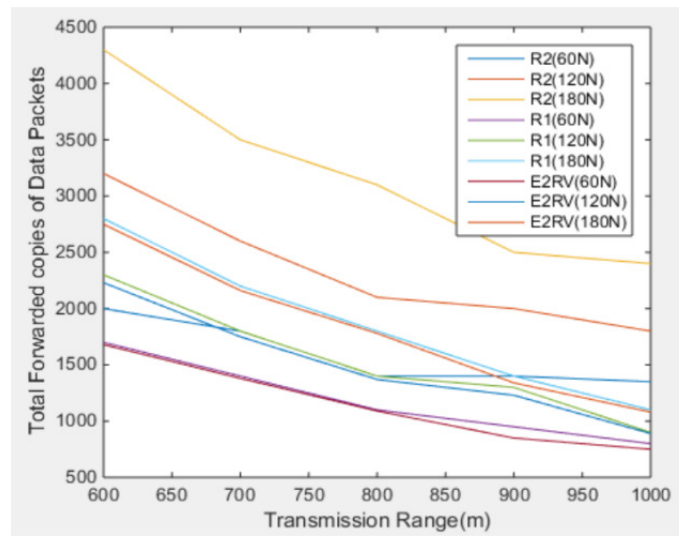

Figure 4: Transmission Range v/s Total number of broadcasted copies 


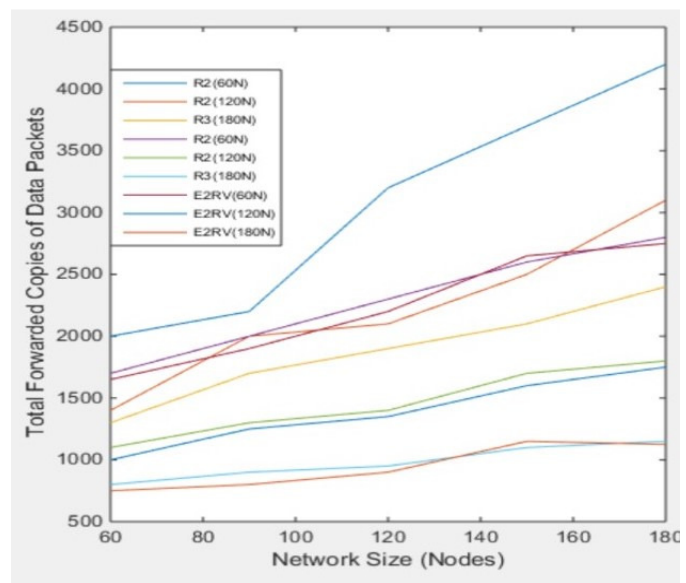

Figure 5: Network Size v/s Total number of forwarded copies of Data packets

Another observation regarding broadcasting copies of data packets is that, forwarded copies of data packets are inversely relative to the broadcast range. It is obvious that when transmission range increases packet suppression increases also the chances for packet collision is also increases. From the simulation results shown in figure 4 is, E2RV is comparing with two algorithms EDOVE [29] called as R1 and WDFAD-DAB [20] called as R2. E2RV is broadcasting lesser copies of data packets and R1 and R2 algorithms. It is approximate 55\% lesser than $\mathrm{R} 2$ and 5\% lesser than $\mathrm{R} 1$ algorithm

\subsection{PDR- Packet Delivery Ratios}

PDR is the total packet reached at the sink node to the whole packets generated by the sender nodes.

$$
\mathrm{PDR}=\frac{\cup_{i=1}^{m} s i}{n}
$$

Here $\mathrm{n}$ is the whole number of packets generated. $\mathrm{m}$ is the total number of sinks. PDR can also be defined as the whole number of packets reached by all the sinks to the whole number of nodes.

The objective of most of the steering algorithm is to improve the data packet delivery ratio. As we know, the number of data packets generated is directly proportional to the network size. When the packet generation rate is high, chances of a collision is increased. Also the relaying nodes exhaust soon because of its energy usage in forwarding the data packets. E2RV is selected the forwarding node by computing the holding time of all the nodes. In this way PDR can be improved. Dead nodes in the network are also increases the void region in the network area which results in the drastic decrease in the PDR. Figure 6 shows the PDR with respect to network size for E2RV algorithm. It shows that PDR slightly increases with the increase in network size or remains constant or. 
International Journal of Computer Networks \& Communications (IJCNC) Vol.10, No.4, July 2018

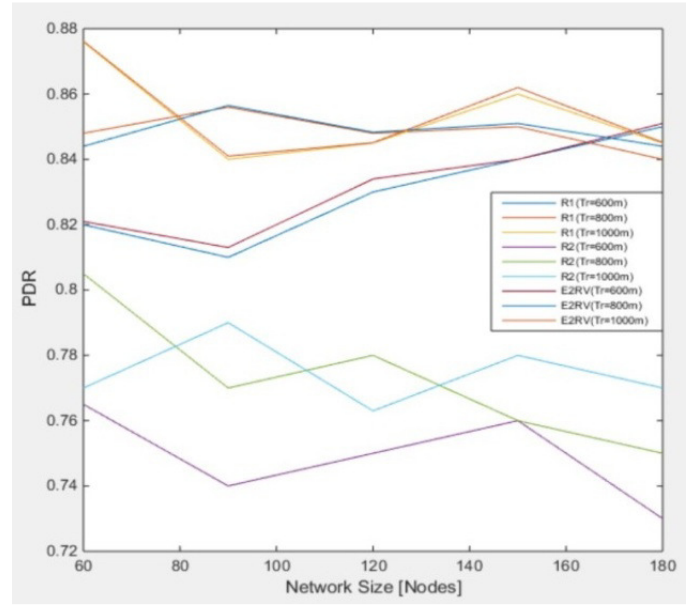

Figure 6: Packet to delivery Ration v/s Network Size

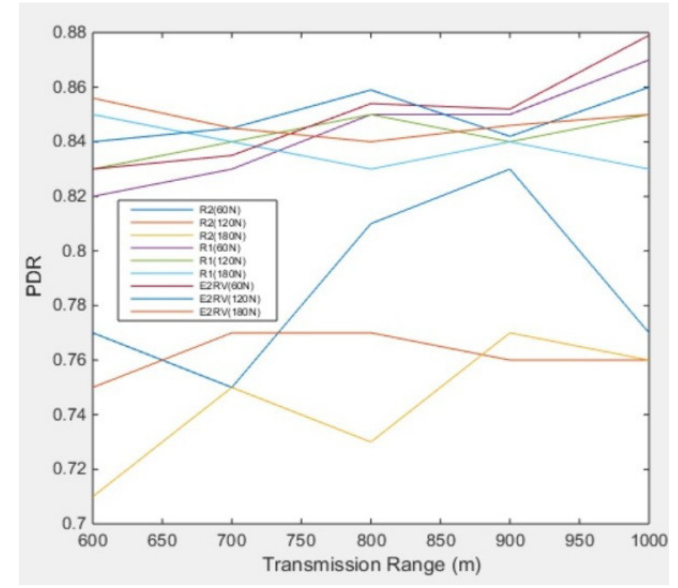

Figure 7: Packet to delivery Ration v/s Broadcast Range

Next graph is between PDR and transmission range. It is shown in figure 7 that as the transmission range increase PDR is also increased as the forwarder nodes can be decreased. This will increase the network lifetime as we decrease the intermediate hops also does not create the void region immediately.

\subsection{Energy Consumption}

The total energy consumption is the sum of transmission energy, receiving energy and idle energy. Figure 8 shows the network energy consumption for various network dimensions for the fixed transmission's range. It seems very clear from the chart shown below that the overall energy consumption is directly proportional to the size of the network. As the size of the network increases energy consumption is also increases. 
International Journal of Computer Networks \& Communications (IJCNC) Vol.10, No.4, July 2018

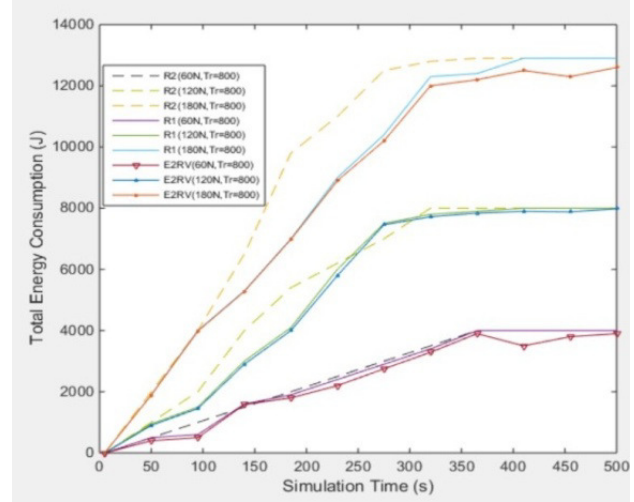

Figure 8: Total Energy Consumption vs Simulation Time

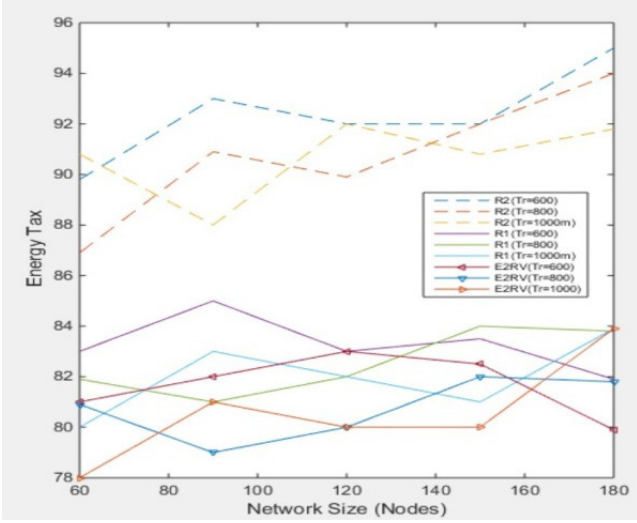

Figure 9: Energy Tax vs Network Size

Figure 9 and 10 is showing the graph for total energy consumption for the fixed network size and varying transmission range. It shows that the energy of E2RV is less than other algorithms for varying network sizes. In addition, energy tax for various algorithms increases with the increase in network size. Figure 10 is showing that there is not any impact of changing the transmission range on energy consumption. Also after a particular transmission time all the algorithms give constant energy consumption because at that time void regions are created due to dead nodes of the network. This means that no more data forwarding is possible through this.

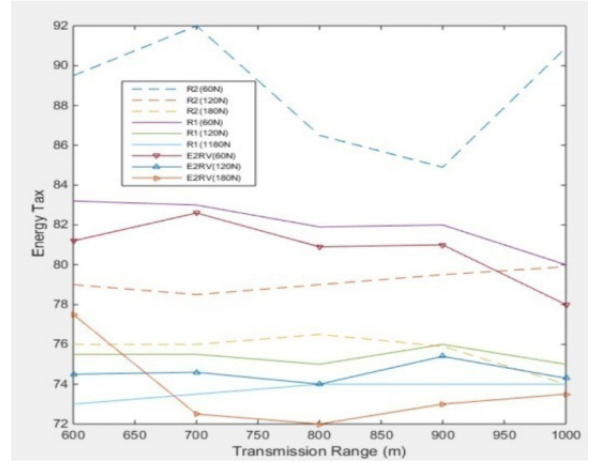

Figure 10: Energy Tax v/s Transmission Range 


\subsection{Number of dead nodes}

A dead node is that node whose battery power is completely used and has no power to perform any task. A number of the dead node is directly proportional to the network size, as network size increases the number of dead node increases. With increased network size number of forwarder node increases which results in the depletion of energy. So it is clearly shown in the chart shown below that with the increase in the size of the network number of dead nodes increasing. Figure 11 depicts the average number of dead nodes to the size of networks. Also, figure 12 shows that no of dead node is less than the R1 and R2 in various network sizes.

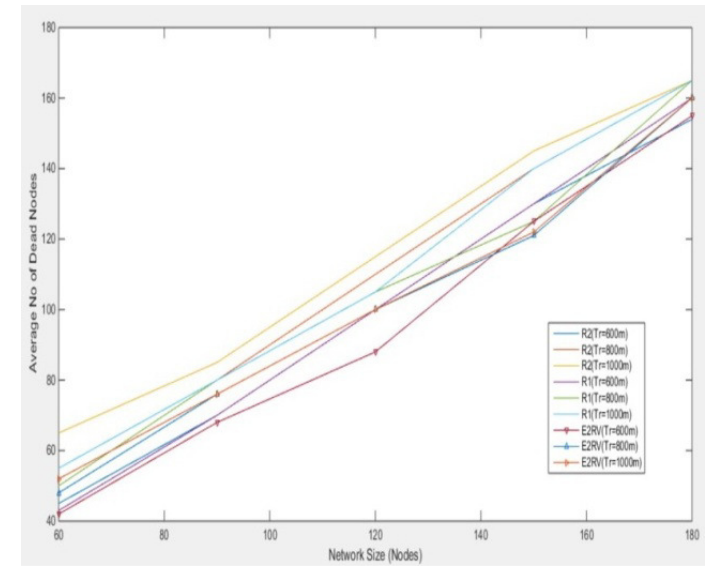

Figure 11: Average number of dead nodes with respect to the Network Size.

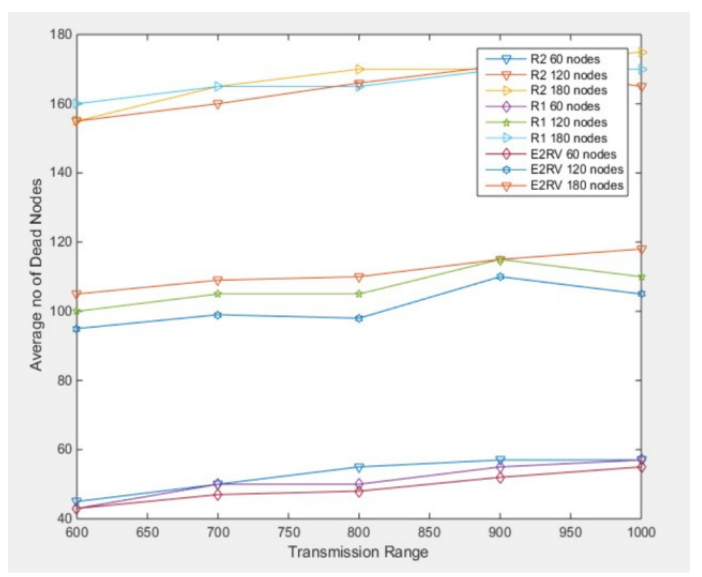

Figure 12: Average number of dead nodes with varying transmission range.

It is shown from the figure 12 that shows that the average number of the dead nodes increases as the network size increases, which are only because the data packet traffic drastically increases with the increase in the network size.

\subsection{Average Operational Time}

The total simulation time or average operational time is defined as the time instance at which the last packet received by the sink node, that is sent by the sender node. 
International Journal of Computer Networks \& Communications (IJCNC) Vol.10, No.4, July 2018

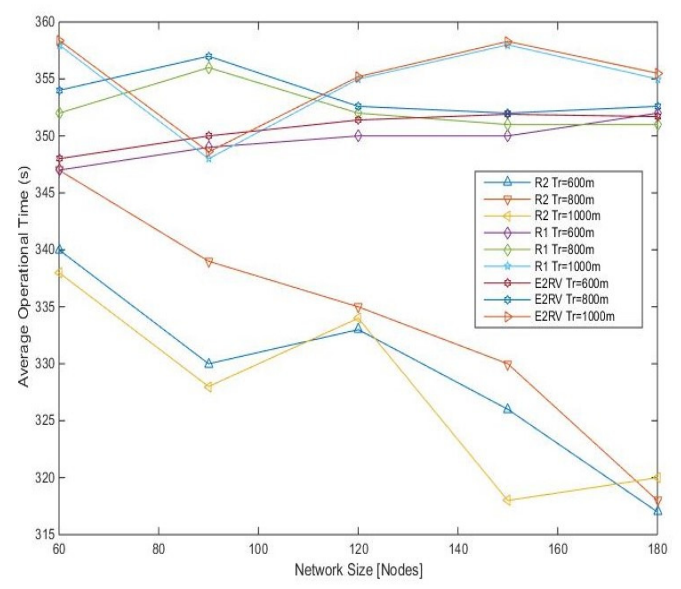

Figure 13: Average Operational Time with respect to the Network Size

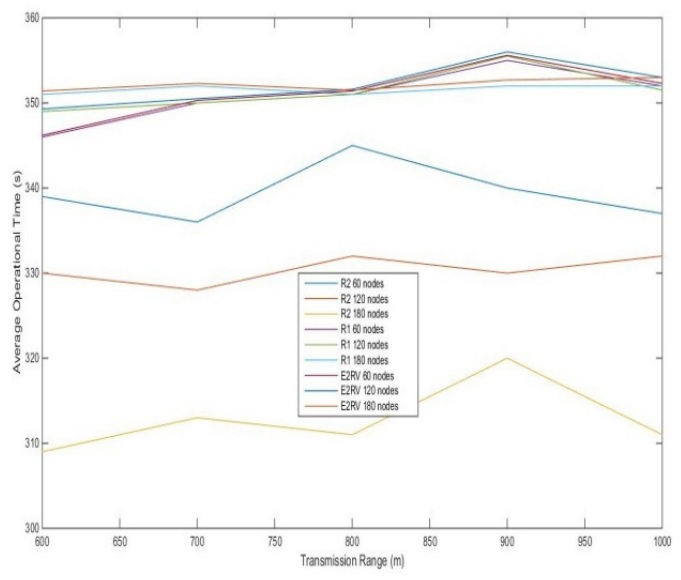

Figure 14: Average Operational Time v/s Transmission Range

Figure 13 shows the results for the average operational time vs. network size. It is clear from the figure that E2RV is having high operational time than previous algorithms. In addition, it depicts from the figure that E2RV have almost stable equipped time as per the network size.

Figure 14 shows the average number of hops for different sized networks and transmission ranges. It is shown that E2RV have the higher number of hops than R1 and R2 it is just because E2RV considers various factors (residual energy, depth difference, the variance of neighbouring nodes depths) for energy expenditure and increase the network lifespan. 


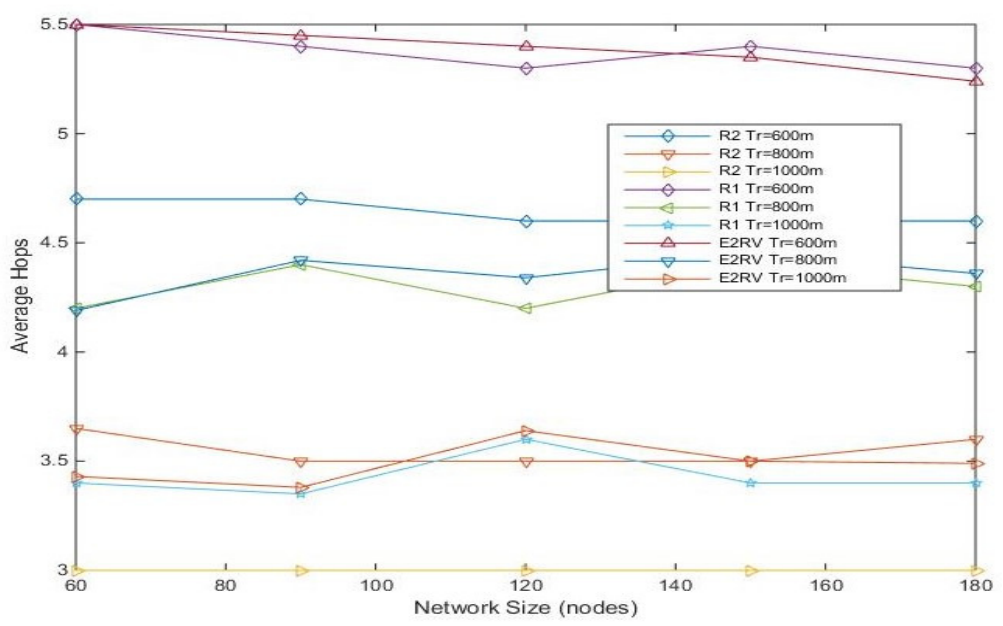

Figure 15: Average number of Hops vs Network Size

So, the proposed algorithm E2RV scheme provides the improved packet to delivery ration, preserve the more energy to increase the network lifetime. It is having higher operational time and higher propagation delay.

\section{CONCLUSION}

This paper proposed an Energy-efficient routing protocol for void avoidance. It uses the deepness variance information between the source node and its one and two hop neighbours. After getting the depth difference, it finds the best suitable forwarder by calculating the normalized remaining energy of nodules and also it calculates normalized deepness discrepancy of two hop forwarder nodes to calculate the holding time of the packet by a particular node. Like this E2RV reduces the overall energy expenditure, increases the lifetime of the network by distributing the load over nodes equally. Various result graphs in section 4 shown that the E2RV performs better in terms of high packet delivery ration, lesser duplicate values, increases lifetime, lesser energy consumption. It is having a high delay, which is the common factor for UWSN.

\section{REFERENCES}

[1] Garcia, J.E. 2005, Accurate positioning for underwater acoustic networks, In Proceedings of the 2005-Europe Oceans, Brest, France.

[2] Sozer, E.M.; Stojanovic, M.; Proakis, 2000, J.G. Underwater acoustic networks. IEEE J. Ocean. Eng., $25,72-83$.

[3] Stojanovic, M.; Preisig, J. 2009 Underwater acoustic communication channels: Propagation models and statistical characterization. IEEE Commun. Mag., 47, 84-89.

[4] Che, X.; Wells, I.; Dickers, G.; Kear, P.; Gong, X. 2010, Re-evaluation of RF electromagnetic communication in underwater sensor networks. IEEE Commun. Mag., 48, 143-151.

[5] Cella, U.M.; Johnstone, R.; Shuley, N. 2009, Electromagnetic wave wireless communication in shallow water coastal environment: Theoretical analysis and experimental results. In Proceedings of the Fourth ACM International Workshop on Underwater Networks, Berkeley, CA, USA.

[6] Kaushal, H.; Kaddoum, G. 2016, Underwater optical wireless communication. IEEE Access, 4, 15181547. 
International Journal of Computer Networks \& Communications (IJCNC) Vol.10, No.4, July 2018

[7] Hanson, F.; Radic, S. 2008, High bandwidth underwater optical communication. Appl. Opt., 47, 277283.

[8] Heidemann, J.; Ye, W.; Wills, J.; Syed, A.; Li, Y. 2006, Research challenges and applications for underwater sensor networking. In Proceedings of the IEEE Wireless Communications and Networking Conference, Las Vegas, NV, USA.

[9] Akyildiz, I.F.; Pompili, D.; Melodia, T. Underwater acoustic sensor networks: Research challenges. Ad Hoc Netw. 2005, 3, 257-279.

[10] Xie, P.; Cui, J.-H.; Lao, L. VBF: Vector-based forwarding protocol for underwater sensor networks. In Proceedings of the 5th International IFIP-TC6 conference on Networking Technologies, Services, and Protocols, Performance of Computer and Communication Networks, Mobile and Wireless Communications Systems, Coimbra, Portugal, 15-19 May 2006.

[11] Nicolaou, N.; See, A.; Xie, P.; Cui, J.H.; Maggiorini, D. Improving the robustness of location-based routing for underwater sensor networks. In Proceedings of the 2007-Europe Oceans, Aberdeen, UK, 18-21 June 2007.

[12] Hwang, D.; Kim, D. DFR: Directional flooding-based routing protocol for underwater sensor networks. In Proceedings of the OCEANS 2008, Quebec City, QC, Canada, 15-18 September 2008.

[13] Ali, T.; Jung, L.T.; Ameer, S. Flooding control by using Angle Based Cone for UWSNs. In Proceedings of the 2012 International Symposium on Telecommunication Technologies, Kuala Lumpur, Malaysia, 26-28 November 2012.

[14] Yu, H.; Yao, N.; Liu, J. An adaptive routing protocol in underwater sparse acoustic sensor networks. Ad Hoc Netw. 2015, 34, 121-143.

[15] Yan, H.; Shi, Z.J.; Cui, J.-H. DBR: Depth-based routing for underwater sensor networks. In Proceedings of the 7th International IFIP-TC6 Networking Conference on Ad Hoc and Sensor Networks, Wireless Networks, Next Generation Internet, Singapore, 5-9 May 2008.

[16] Wahid, A.; Lee, S.; Jeong, H.-J.; Kim, D. EEDBR: Energy-efficient depth-based routing protocol for underwater wireless sensor networks. In Advanced Computer Science and Information Technology; Springer-Verlag: Berlin/Heidelberg, Germany, 2011; pp. 223-234.

[17] Barbeau, M.; Blouin, S.; Cervera, G.; Garcia-Alfaro, J.; Kranakis, E. Location-free link state routing for underwater acoustic sensor networks. In Proceedings of the 28th IEEE Canadian Conference on Electrical and Computer Engineering (CCECE), Halifax, NS, Canada, 3-6 May 2015.

[18] Ghoreyshi, S.M.; Shahrabi, A.; Boutaleb, T. An inherently void avoidance routing protocol for underwater sensor networks. In Proceedings of the Twelfth IEEE International Symposium on Wireless Communication Systems, Brussels, Belgium, 25-28 August 2015.

[19] Ghoreyshi, S.M.; Shahrabi, A.; Boutaleb, T. A novel cooperative opportunistic routing scheme for underwater sensor networks. Sensors 2016, 16, 297.

[20] Yu, H.; Yao, N.; Wang, T.; Li, G.; Gao, Z.; Tan, G. WDFAD-DBR: Weighting depth and forwarding area division DBR routing protocol for uasns. Ad Hoc Netw. 2016, 37, 256-282.

[21] Han, G.; Jiang, J.; Bao, N.; Wan, L.; Guizani, M. Routing protocols for underwater wireless sensor networks. IEEE Commun. Mag. 2015, 53, 72-78.

[22] Li, N.; Martínez, J.-F.; Meneses Chaus, J.M.; Eckert, M. A survey on underwater acoustic sensor network routing protocols. Sensors 2016, 16, 414.

[23] Ghoreyshi, S.M.; Shahrabi, A.; Boutaleb, T. Void-handling techniques for routing protocols in underwater sensor networks: Survey and challenges. IEEE Commun. Surv. Tutor. 2017, 19, 800-827. 
[24] Otnes, R.; Haavik, S. Duplicate reduction with adaptive backoff for a flooding-based underwater network protocol. In Proceedings of the 2013 MTS/IEEE OCEANS, Bergen, Norway, 10-14 June 2013.

[25] Komulainen, A.; Nilsson, J. Capacity improvements for reduced flooding using distance to sink information in underwater networks. In Proceedings of the 2014 Underwater Communications and Networking (UComms), Sestri Levante, Italy, 3-5 September 2014.

[26] Al-Karaki, Jamal. and E. Kamal, Ahmed.,. "Routing Techniques in Wireless Sensor Networks: A Survey", IEEE Communications Magazine, vol 11, no. 6, pp. 6-28, Dec 2004.

[27] Abdul Wahid, "MRP: A Localization free Multi layered routing algorithm for Underwater Wireless Sensor Networks", Volume 77, Issue 4, pp 2997-3012, August 2014.

[28] G. Liu and Z. Li, "Depth-based multi-hop routing algorithm for underwater sensor network," in Proceedings of the 2nd International Conference on Industrial Mechatronics and Automation (ICIMA '10), pp. 268-270, IEEE, May 2010.

[29] Safdar Hussain Bouk, Syed Hassan Ahmed, Kyung-Joon Park and Yongsoon Eun, "EDOVE: Energy and Depth Variance-Based Opportunistic Void Avoidance Scheme forUnderwater Acoustic Sensor Networks", Sensors 2017, 17, 2212; doi: 10.3390/s17102212, pp 1-25.

\section{AUTHORS}

Gulista Khan born in India on September, 30, 1985. She completed her B.Tech. in Information technology from Shri Krishan Institute of Engineering And Technology, Kurukshetra in 2006. She completed Post Graduation (M. Tech.) from MMEC,Mullana in 2009. She is pursuing Ph.D. in Computer Science and Engineering. Her area of specialization is Wireless Sensor Network. In past she has worked as Lecturer in Haryana Engineering College,Jagadhri, for 5 years. Currently working as Assistant Professor in

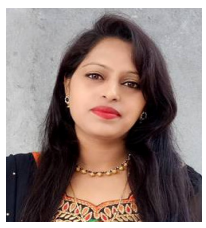
Computer Science and Engineering department, Teerthanker Mahaveer University.

Dr. Rakesh Kumar Dwivedi is M.Tech. in Computer Science and Engineering from H.B.T.I. Kanpur, Uttar Pradesh and Ph.D. in the area of Digital Image Processing from Indian Institute of Technology Roorkee, Roorkee. He has more than 18 years of teaching experience. Published 11 papers in International Journals of high impact factor and 38 research papers in Conferences in India and Abroad. Chaired the session in the World Congress of Computer Science and Computer Engineering conference, 2014 at Las Vegas, USA. He participated in 17 AICTE approved short term courses in the area of

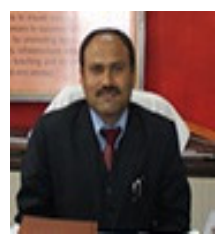
Computer Science. His research interest includes the soft computing; Fuzzy based Hybrid Soft Classification, Parameter Optimization, and Algorithm design and uncertainty reduction using fuzzy techniques, Wireless Sensor Network. Currently working as Principal and Professor in CCSIT, Teerthanker Mahaveer University, Moradabad, India 